\title{
HIGH THROUGHPUT SOLID PHASE MICROEXTRACTION: A NEW ALTERNATIVE FOR ANALYSIS OF CELULAR LIPIDOME?
}

\author{
Afsoon Pajand Birjandi', Barbara Bojko" ${ }^{1 \#}$, Zhibin Ning², Daniel Figeys², Janusz \\ Pawliszyn ${ }^{1 *}$ \\ ${ }^{1}$ Department of Chemistry, University of Waterloo, 200 University Avenue West, Waterloo, \\ Ontario, N2L 3G1, Canada \\ ${ }^{2}$ Ottawa Institute of Systems Biology, University of Ottawa, 451 Smyth Road, Ottawa, ON K1H \\ 8M5, Canada
}

*Corresponding author. Tel.: +1-519-888-4641; Fax: +1-519-746-0435

E-mail address: janusz@uwaterloo.ca

\#Present address: Department of Pharmacodynamics and Molecular Pharmacology, Faculty of Pharmacy, Collegium Medicum in Bydgoszcz, Nicolaus Copenicus University in Torun, dr A. Jurasza 2, 85-089 Bydgoszcz, Poland 


\title{
HIGH THROUGHPUT SOLID PHASE MICROEXTRACTION: A NEW ALTERNATIVE FOR ANALYSIS OF CELLLAR LIPIDOME?
}

\author{
Afsoon Pajand Birjandi†, Barbara Bojko†, Zhibin Ning $¥$, Daniel Figeys $¥$, Janusz Pawliszyn†* \\ † Department of Chemistry, University of Waterloo, 200 University Avenue West, Waterloo, Ontario, N2L 3G1, Canada \\ ‡ Ottawa Institute of Systems Biology, University of Ottawa, Ontario, Canada
}

ABSTRACT: A new SPME method for untargeted lipidomic study of cell line cultures was proposed for the first time. In this study the feasibility to monitor changes in lipid profile after external stimuli was demonstrated and compared to the conventional Bligh \& Dyer method. The human hepatocellular carcinoma (HCC) cell line was used as a model. The obtained results provided a list of up-regulated and down-regulated lipids through a comparison between control (non-stimulated) cells versus the group of cells treated with polyunsaturated fatty acid (20:5). Use of the SPME technique yielded a list of 77 lipid species whose concentrations were recognized to be significantly different between control and treated cells, from which 63 lipids were up-regulated in treated cells. In general, the list was comparable to the peer list obtained by the Bligh \& Dyer method. However, more diversity of lipid classes and subclasses such as LPC, sphingomyelins, ceramides, and prenol lipids were observed with the application of the SPME method. Method precision for the SPME approach was within the acceptable analytical range (5-18\% RSD) for all detected lipids, which was advantageous over solvent extraction applied. The evaluation of ionization efficiency indicated no matrix effect for the SPME technique, while Bligh \& Dyer presented significant ionization suppression for low abundant species such as LysoPC, PG, ceramides, and sphingomyelins, and ionization enhancement for high abundant phospholipids such as PE.

Keywords: lipidomics, cell line study, solid phase microextraction, automation 


\section{Introduction}

For the past few years, lipids, and in particular omega- 3 and omega- 6 polyunsaturated fatty acids (PUFAs) have received considerable attention in human health. Epidemiological studies are trying to find evidences for possible correlation between PUFA-rich diets and a reduced incidence of cancer [1-4]. These findings could be examined and validated through "discovery lipidomics" which is a new branch of lipidomic studies $[5,6]$. In order to obtain reliable and meaningful answers in lipid discovery experiments, not only specialized mass spectrometric techniques are required, reliable sample preparation strategies are also needed before implementation of the instrumental procedure. In this regard, classical sample extraction methods have not been progressive enough to attain the quick advancements in state-of-theart mass spectrometry technologies; consequently, sample preparation techniques have become the weakest link in modern analytical procedures [7,8]. Nevertheless, conventional sample preparation methods that rely on manual lipid extraction approaches are unable to fulfill the need for large scale lipidomics studies that require analysis of numerous samples per day. Therefore, automation of the sample preparation and extraction procedures is essential for improving the time/cost effectiveness of such projects, minimizing sample losses, and avoiding unexpected experimental artifacts. Traditional liquid-liquid extraction (LLE) approaches such as Bligh \& Dyer [9] or Folch [10] protocols, have been commonly used for lipids analysis based on organic solvent extraction. Over the past years, attempts have been made to boost the robot-assisted sample preparation and lipid extraction procedure based on LLE in the high throughput format [11-15]. However, the LLE method is burdened by a large solvent-to-sample ratio that besides toxicity, it also poses challenges for automation in the 96well format. To bypass these difficulties, automated 96-well format solid-phase extraction (SPE) has been widely used for high-throughput determination of phospholipids in nutritional and epidemiological studies [16-17]. Plenty of sorbent chemistries such as aminopropyl silica, mixed mode, and C18, have been applied towards the analysis of lipids; however, these studies have either focused mainly on fatty acids, [18-19] or were otherwise class-selective depending on the sorbent and elution conditions used [20-26]. Moreover, several attempts have been made to combine liquid-liquid extraction and solid phase extraction methods (LLE-SPE) for plasma and blood, primarily with the purpose of separating phospholipids from the rest of the metabolites, resulting in the achievement of better metabolome coverage [24,27].

Recently, a novel application of the solid-phase microextraction (SPME) was presented for the analysis of free (unconjugated) fatty acids from biological matrices [28]. However, in spite of 
the promising potential of this technique for the extraction of hydrophilic and hydrophobic compounds, it has never been applied for the analysis of lipid and lipidomics studies. Thin film microextraction (TF-SPME) as a relatively newly emerged format of SPME offers improvement of sensitivity without sacrificing time by increasing the available surface area and volume of coatings. The current configurations of TF-SPME are available on fully automated 96-well plate system and since its inception, different coating chemistries have been reported for various applications including analysis of drugs and metabolites from biofluids, tissue, cells, and food matrices [29-40]. The present study is an attempt to design and develop a high throughput SPME application for the lipidomics studies and compare the obtained results with selected standard approach based on solvent extraction thus evaluate feasibility of the proposed solution for the given type of analysis. To perform the analysis the human hepatocellular carcinoma (HCC) cell line was used as a model. In order to asses the ability of the method to monitor biological changes occurring in the studied system the cancer cells were supplemented with chosen PUFA, because it was reported before that it may influence phospholipid fraction of cancer cells $[3,41]$. This modification alters the lipid metabolism pattern and consequently interrupts the lipid bilayer membrane, also changes the cell morphology, and suppress cell growth. The current study considered these findings to investigate lipidome alterations in treated cells while comparing the proposed SPME method to the conventional LLE methodology based on the Bligh \& Dyer protocol. Using this module, we aimed to evaluate the strengths and weaknesses of the validated SPME protocol in providing a comprehensive profile of lipidome changes.

\section{Materials and methods}




\subsection{Cell culture}

Human HCC cell line Huh7 was seeded in six $75 \mathrm{~cm} 2$ flasks $(n=3)$ in DMEM supplemented with $10 \%$ fetal bovine serum, $2 \mathrm{mM} \mathrm{L-glutamine,} \mathrm{and} \mathrm{penicillin/streptomycin,} \mathrm{then} \mathrm{incubated} \mathrm{at} 37^{\circ} \mathrm{C}$ in a humidified $\mathrm{CO} 2$ incubator. Twenty four hours after seeding, media was changed to serumfree medium for 6hrs; three flasks containing $\mathrm{HUH7}$ cells were then treated with eicosapentanoic acid (EPA) at $0.4 \mathrm{mM}$ for $4 \mathrm{hrs}$, while the control set $(n=3)$ was kept untreated. Cells were then washed with $1 \mathrm{ml}$ of cold phosphate-buffered saline (PBS) twice, and scraped off the surface of the flask using a cell scraper in PBS.

The cell suspension was then spun at $5000 \times \mathrm{g}$ for $20 \mathrm{~min}$, the supernatant discarded, and the cell pellet flash-frozen in liquid nitrogen. The pellets were stored at $-80^{\circ} \mathrm{C}$ until use. Each cell pellet of biological replicates ( $n=3$ control and $n=3$ treated) contained approximately $8.4 \times 106$ HUH7 cells. Upon analysis, samples were thawed at room temperature, and $6 \mathrm{ml}$ of cold PBS were added to each sample tube, which were gently swirled until complete suspension was achieved. The content of each tube was divided into six aliquots of $1 \mathrm{ml}$, from which $2 \times 1 \mathrm{ml}$ portions were subjected to the lipid extraction based on Bligh \& Dyer procedure, and $4 \times 1 \mathrm{ml}$ subjected to SPME analysis.

\subsection{Lipid extraction by Bligh \& Dyer protocol}

The standard procedure of lipid extraction was performed according to the Bligh \& Dyer method [42]. The total lipid fraction was extracted from $1 \mathrm{ml}$ cell suspension $(1.70 \times 106 \mathrm{cells} / \mathrm{ml})$ with the addition of $3.75 \mathrm{ml}$ chloroform/MeOH mixture $(2: 1, \mathrm{v} / \mathrm{v})$, followed by vigorous shaking. Then, $1.25 \mathrm{ml}$ extra chloroform was added to the mixture solution and vortexed for $20 \mathrm{~s}$, followed by addition of $1.25 \mathrm{ml}$ nanopure water while swirling to form a two-phase system. The mixture then was settled at room temperature for $10 \mathrm{~min}$ to reach equilibration, and subsequently centrifuged at $2000 \mathrm{RPM}$ in $5{ }^{\circ} \mathrm{C}$ for $5 \mathrm{~min}$. The organic bottom phase was transferred to a new tube and evaporated under N2 gas to dryness following by reconstitution in $1 \mathrm{ml} \mathrm{IPA/methanol} \mathrm{(1:1)} \mathrm{before} \mathrm{injection} \mathrm{to} \mathrm{LCMS.} \mathrm{For} \mathrm{each} \mathrm{method,} \mathrm{preparation} \mathrm{and}$ extraction of cell samples were carried out separately. In order to avoid bias, sample preparation was carried in a randomized order for biological replicates.

\subsection{SPME procedure}

A detailed description of the preparation of the C18-PAN 96-blade coatings is provided in Section 2 of the Appendix [43]. SPME coatings were pre-conditioned for $30 \mathrm{~min}$ in a mixture of methanol/water (1:1) prior to use. For the SPME approach, cells were homogenized by vigorous agitation using the VWR DVX-2500 digital vortex (Mississauga, ON, Canada) and incubated for 
$30 \mathrm{~min}$ at $37^{\circ} \mathrm{C}$ prior to analysis. The SPME blade assembly were directly immersed into $1 \mathrm{~mL}$ sample aliquots pipetted into 96-deep well plate, and agitated with $2400 \mathrm{rpm}$ vortex for a total extraction time of 90 minute. Immediately after extraction, coatings were rinsed by dipping in purified water (18.2 M $\Omega$ ) for $10 \mathrm{~s}$ to remove any residuals of biological material. Subsequently, thin-film coatings were agitated for 60 minutes in $1 \mathrm{ml}$ IPA/methanol (1:1 v/v) for proper desorption of lipids, and the resulting final extract solutions injected to the HPLC-ESI-MS/MS system for analysis. The total workflow from sampling to sample preparation is presented in Figure S-1 of Appendix.

\subsection{LC-ESI-MS operating conditions}

Analyses of cell extractions were performed on a LC-MS system consisting of the Accela autosampler with a cooled system tray, Accela LC Pumps, and the Exactive Orbitrap mass spectrometer (Thermo, San Jose, CA, USA) equipped with electrospray ionization (ESI) probe. To maintain a mass accuracy better than $5 \mathrm{ppm}$, the Exactive Benchtop Orbitrap was calibrated every $24 \mathrm{hr}$ with a Thermo Scientific ESI Calibration Solution. Samples were analyzed with the use of an XBridge BEH C18, $2.5 \mu \mathrm{m}, 150 \mathrm{~mm} \times 2.1 \mathrm{~mm}$ column XP (Waters Corp, Milford, MA). A binary solvent system was used for gradient elution that consisted of water/acetonitrile (1:9), with $0.1 \%$ acetic acid as mobile phase $A$ and isopropyl alcohol as mobile phase $B$, and $0.15 \mathrm{mM}$ ammonium acetate, for a constant flow rate of $200 \mu \mathrm{l} / \mathrm{min}$. The instrumental conditions are further described in Section 5 of the Appendix.

\subsection{Data processing}

The msConvertGUI.exe program from ProteoWizard was used to convert raw data output to the open .mzXML format [44]. Data were then further processed with the open source XCMS Rpackage (Scripps Center for Metabolomics, CA, USA) for automated retention time correction, molecular feature normalization, peak alignment, and peak integration. Following data processing, ion annotation was conducted with the use of the CAMERA R-package (Bioconductor Version 2.10) to identify adducts and isotopes through application of optimal parameter settings suggested by Patti et al. [44]. After data processing, an exported csv file was generated and converted to a Microsoft Excel spreadsheet containing accurate mass and retention time, $\mathrm{m} / \mathrm{z}$, and related signal intensities.

\subsection{Data analysis}

The produced datasheet was submitted into the SIMCA-P software 11.0 (Umetrics, Umea, Sweden), where statistical data analysis and data visualization were carried out. Feature identification was obtained through a mass-based search against three databases: Human 
Metabolome Database (HMDB), METLIN, and LIPID MAPS, with a mass tolerance window of 5 $\mathrm{ppm}$. In order to minimize the possibility of false identification, the retention time of related peaks was compared with the partition coefficient $(\log P)$ of the matching features.

\section{Results and discussion}

\subsection{LCMS method assessment}

Due to the diverse nature of cell lipidome, both positive and negative ionization modes must be employed in the LC/MS profiling methods to achieve comprehensive coverage. Although some phospholipid species are ionized in both polarities, they exhibit a significantly higher sensitivity in positive ion mode. In contrast, phosphoinositol (PI) and phosphoserine (PS) were the phospholipids that were detectable in negative ion mode in higher intensities (Table S-1 Appendix). Figure S-2 in the Appendix represents the XIC chromatogram of lipid standards in positive and negative ion modes. All detected lipid species for both SPME and Bligh \& Dyer methods demonstrate $\mathrm{S} / \mathrm{N}$ ratio of at least 26 or greater, well above a defined limit of detection (LOD) of $\mathrm{S} / \mathrm{N}=3$. It is important to note that although SPME does extract smaller quantity of lipids, it does not substantially compromise the signal-to-noise and dynamic range. Moreover, the trace level lipids in B\&D may not be identified because they are masked by more abundant species, while in SPME they could be still identified because the total lipid extract does not overload chromatograms and ionization source because of the balanced coverage phenomenon [45]. During analysis, lipid standards showed retention time coefficients of variance (CV) of less than $0.03 \%$ when compared within single ionization mode. The integration of XIC peak areas presented CVs of less than $2.1 \%$ in either ionization modes.

\subsection{Comparison of method validity}

Method precision for each sample preparation procedure was determined on the basis of independent extractions from the cultured cell samples (Figure 1). Despite the fact that SPME is non-exhaustive method based on equilibration between the sample and the extraction phase which could be affected by experimental condition changes the mean variability of SPME was below $18 \%$, which is considered satisfactory for analytical method validation criteria [46]. Contrarily, the results obtained for Bligh \& Dyer demonstrate poor precision, mainly for less hydrophobic lipid species with lower LogP values: SM, MG LPCs. This could be related to the possibility of ionization suppression associated with LLE technique. This issue was investigated further under matrix effect studies in this article. 


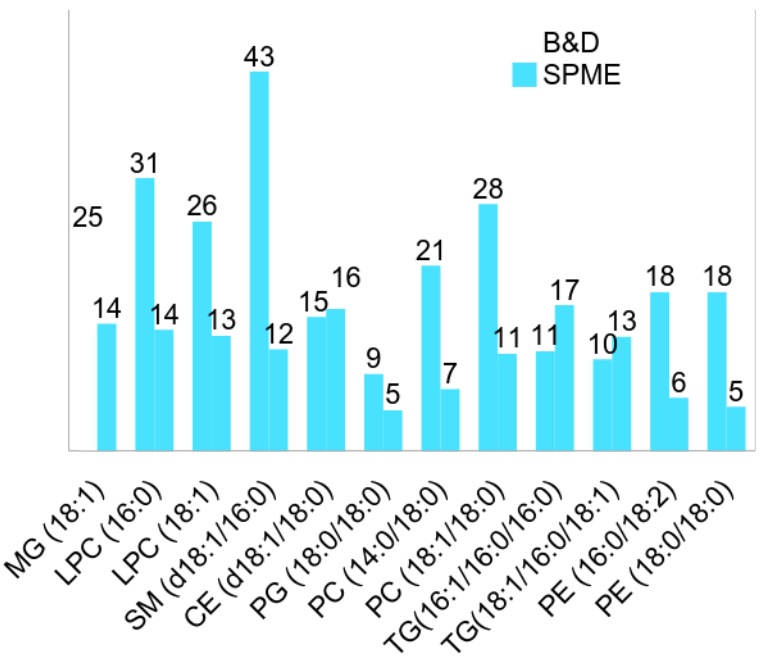

Figure 1

\subsection{Study of ion features acquired by SPME and Bligh \& Dyer}

Analysis of two data sets obtained for SPME indicated a total of 1922 ions in positive and 655 ions in negative mode, while for the Bligh \& Dyer method, 1430 ions were detected in positive and 451 ion in negative mode for a mass range of 100 to $1200 \mathrm{~m} / \mathrm{z}$. Identification of lipids was based on database search for the most probable ion adduct for each lipid category, and at least two adducts at the same retention time for the precise identification assurance. Ion maps and histograms of the distribution of method precision for all unknown features with intensities higher than LOD levels are provided in Figure 2. The ion maps of both methods demonstrate relatively identical coverage of features, yet in general, the metabolite coverage in negative ion mode is less in comparison to coverage obtained for positive ion mode. This is attributed to the better ionization efficiency of lipids in positive ESI mode. For the evaluation of method performance, the absence of analytical variability must be assured [47]. As observed in Figure 2, the distribution of \% RSD for all detected features shows that both extraction methods represent RSDs bellow $30 \%$ in positive ion mode; while for SPME, this value was found to be below $5 \%$ for the majority of peaks. Moreover, in both cases, most features presented RSD values less than $30 \%$ in negative ESI mode, with only a small number of features exceeding $30 \%$ RSD. These data signify that SPME can match and in some cases exceed the precision of the data produced by the conventional method.

\subsection{Statistical analysis}

Principal component analysis (PCA) was conducted on data obtained from HUH7-cell culture analysis, including control and treated cells extracted by both Bligh \& Dyer and SPME methods 
in positive and negative ionization polarities (Figure 3). Orthogonal Partial Least SquaresDiscriminant analysis (OPLS-DA) models were obtained considering the score plots of the first and second components (Figure 4). Data were modeled to visualize discrimination between control and treated cells using SPME and B\&D methods separately. A list was compiled of all the ions that obtain a Variable Importance in the Projection (VIP) values higher than 1 on the score plots, as these ions were driving the difference between groups in the OPLS-DA model. Examples of OPLS-DA plots are illustrated in Figure 3. A detailed discussion about feature identification procedure is presented in the Appendix. Lipids of interest were filtered according to the ANOVA P-value $<0.001$ and fold change $>2$, which decreased the number of lipids of interest. Group separation in the OPLS-DA model between the two extraction methods revealed different strengths and weakness of each method in analysis of lipids, which will be discussed further in this paper.

a) Ion map-Positive

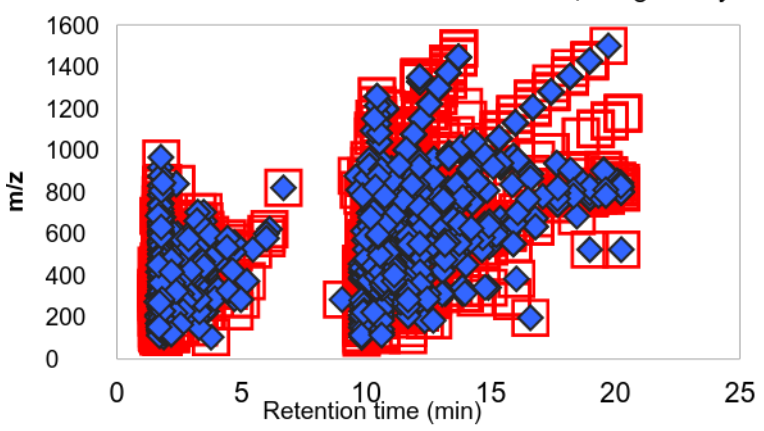

b) Distribution of method \% RSD-Positive mos

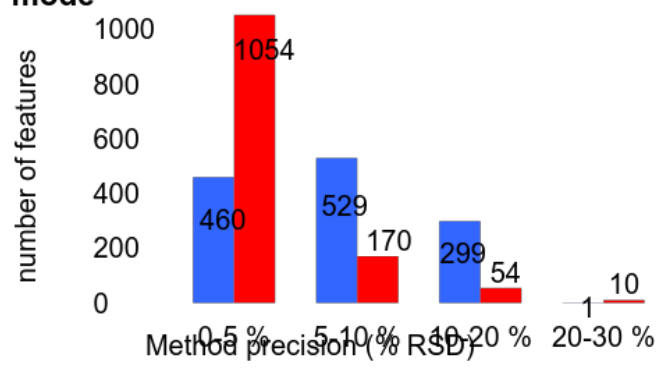

d) Desstribution of method \% RSD-Negative

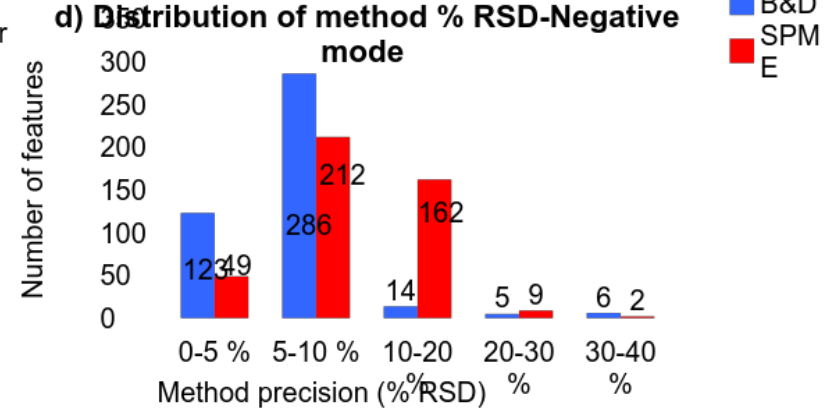

SPME c) Ion map-Negative
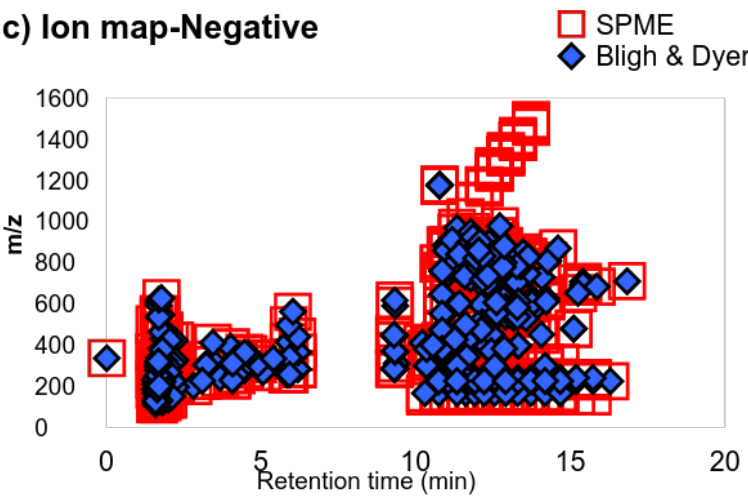

Figure 2 

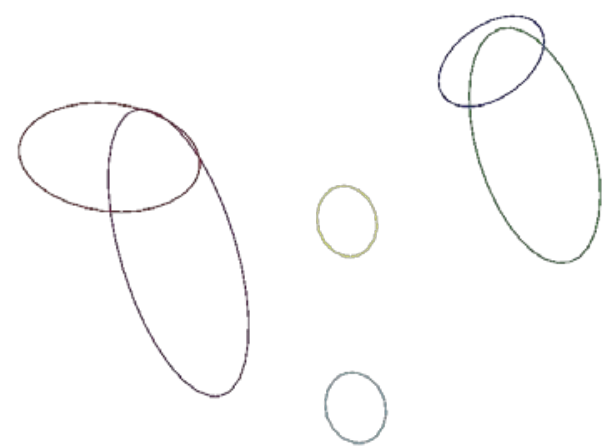

Figure 3

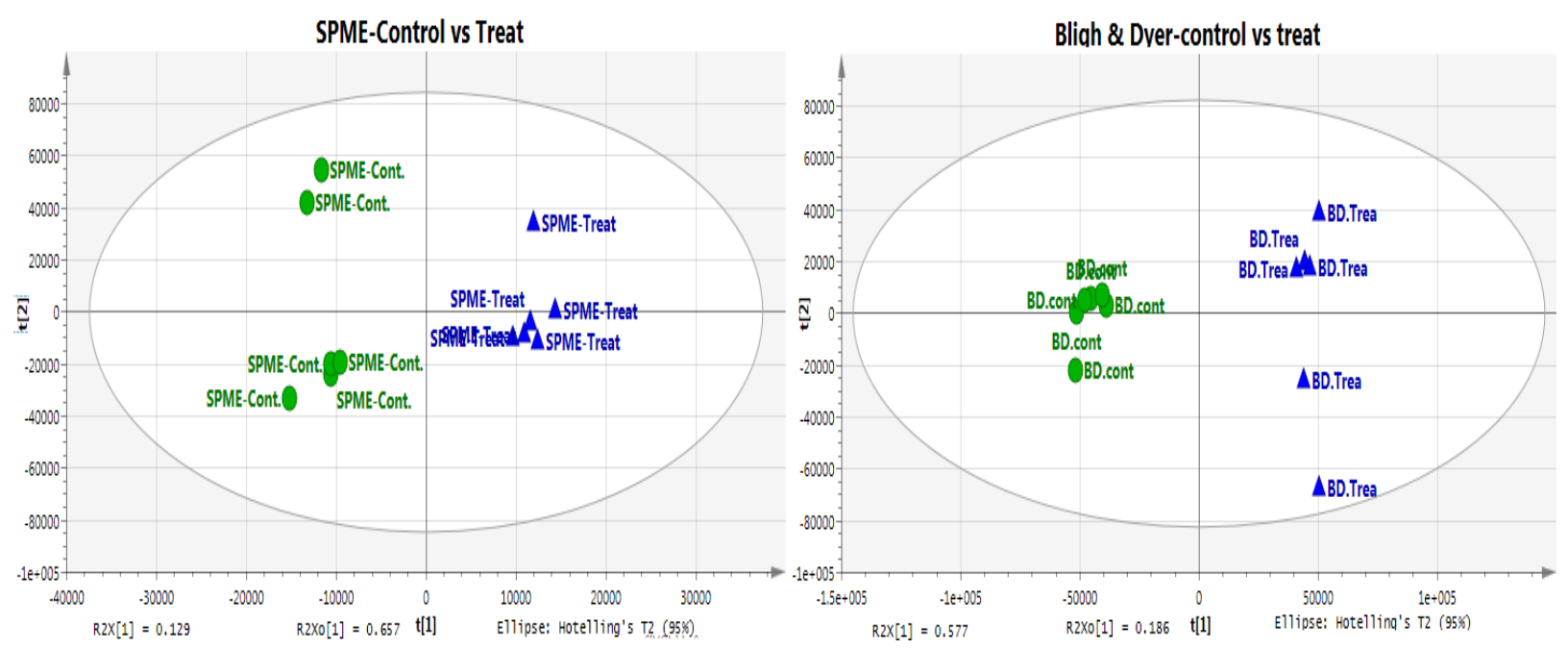

Figure 4

\subsection{Comparison of extraction efficiency of two methods for the group coverage}

The method performance for the coverage of lipid groups/subgroups was compared (Figure 5). Despite the fact that identification of the molecular features was based on mass accuracy and retention time only and no fragmentation pattern was obtained several trends are easily 
apparent. Important finding was extraction of subspecies of the sphingolipid class, which contains ceramides and sphingomyelins, with SPME, but not with B\&D. Moreover, B\&D exhibits a week performance in the extraction of low abundant phospholipids such as LysoPCs and PGs which may be related to ESI saturation. On the other hand, the B\&D method had a distinct advantage for covering a larger number and diversity of high-abundant membrane phospholipids such as PC and PE. Given the high efficiency of chloroform and methanol as extracting solvents in $B \& D$, it is not surprising that higher coverage of membrane phospholipids was observed. This is due to the function of methanol in releasing lipids from their protein-lipid complexes, followed by their complete dissolution in chloroform $[42,48,49]$. In SPME technique, even though samples were vigorously vortexed prior extraction to break down the cell membrane and release intracellular lipidome, the results suggest that the approach did not disintegrate structure of the membrane to the extent of liberating strongly bound membrane lipids. Therefore if membrane lipids are of interest in case of SPME alternative and more aggressive disruption methods e.g. sonication should be considered. Both methods, SPME and $B \& D$ performed similarly in the extraction of triglycerides, although SPME worked better the extraction of less abundant glycerolipids, including monoacylglycerols and diacylglycerols.
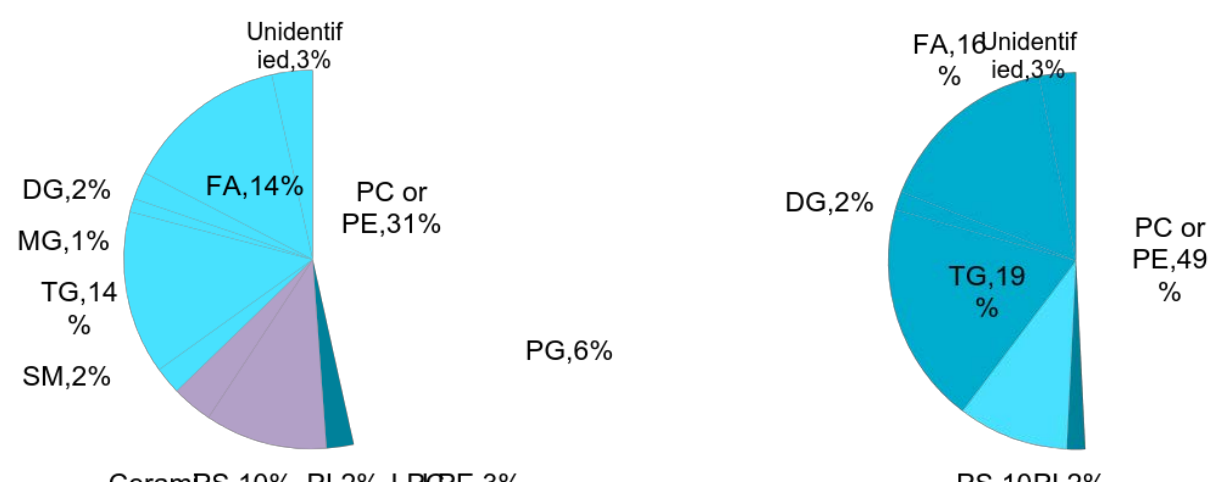

Figure 5

The results implicate that SPME should be considered as a good alternative or a complementary tool for global lipidomics since it shows a better coverage between groups of various lipid categories with the necessity of introducing additional cell disruption step if lipids present only in bound form are of interest. Conversely, Bligh \& Dyer was shown to be highly efficient in the lipidomic study within class-specific lipids of the same (sub) category such as PC or PEs. 


\subsection{Matrix effect evaluation}

Phospholipids among all lipid categories more significantly cause ionization suppression in LCMS approaches, which results in suboptimal ionization of low-abundant lipid species. Absolute matrix effect was assessed using the post extraction spike method according to Matuszewski et al. [50]. ME values larger than $120 \%$ and smaller than $80 \%$ represent significant ionization enhancement or suppression for a given analyte. In the Bligh \& Dyer samples, signal suppression was observed for PG, CE, and LysoPC, while PE and PC indicated significant ionization enhancement (Figure 6). In SPME samples, neither of the lipid species demonstrated a notable matrix effect. This constitutes an advantage of SPME method not only for isolation of lipid analytes from intrusive macromolecules, but also for prevention of the suppressive effect of highly abundant phospholipids in the ESI source. Additionally, the non-exhaustive extraction prevents build-up of lipids on chromatographic column and accumulation in MS system, therefore extending lifetime of the columns and minimizes necessity of frequent maintenance of the instrument.

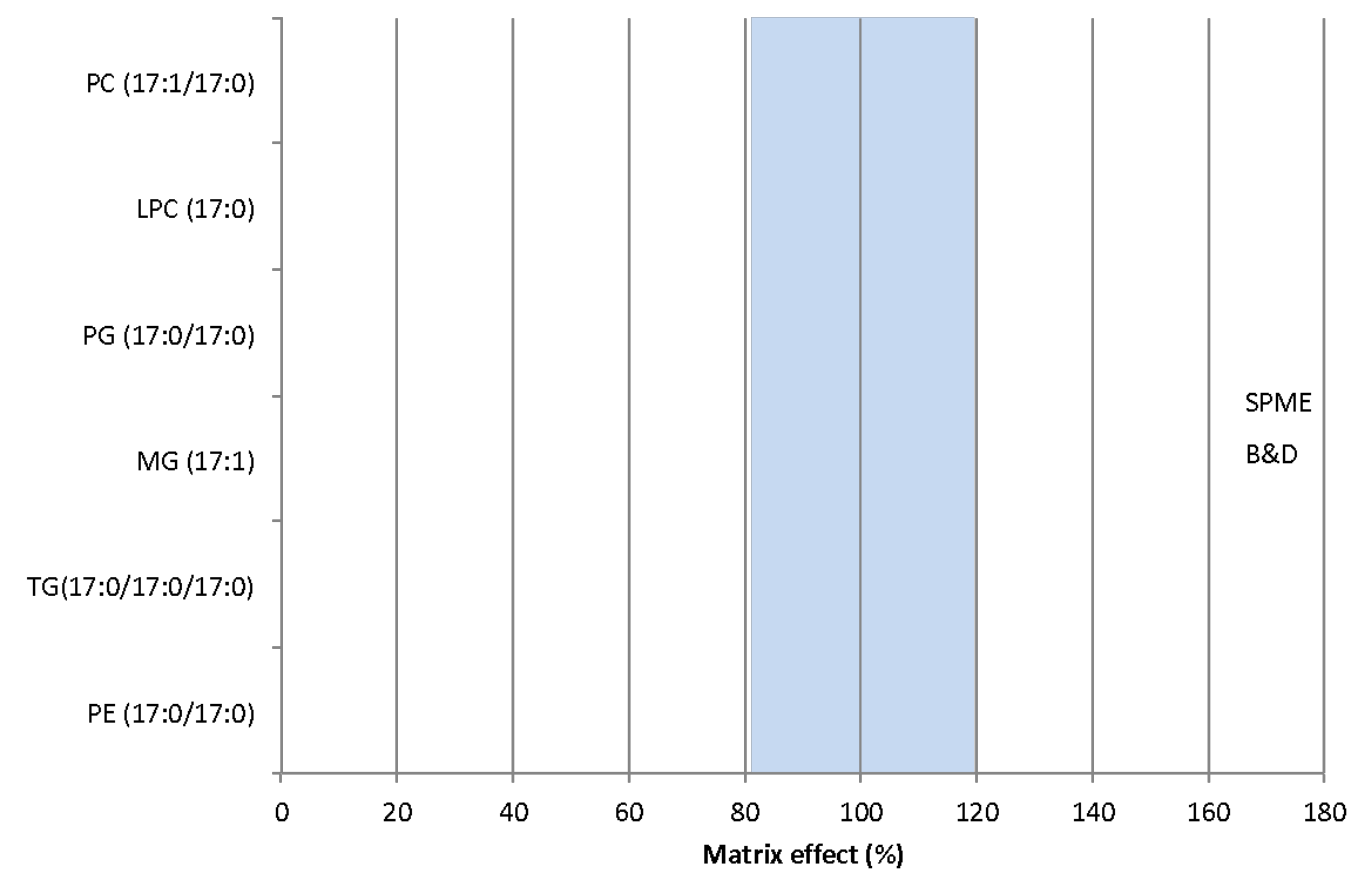

Figure 6

\subsection{Control vs. Treated Cells: Up-regulated lipids}

Following the aforementioned analytical workflow, feature ions were confined to a list of significantly up/down-regulated lipids. It should be emphasized here that the goal of the 
current study was not an in-depth biological interpretation of the EPA influence of the HHC viability per se but assessment of the performance of the proposed protocol and its potential applicability to lipidomics investigation. Considering the lipid ions, which were statistically higher in the group of treated cells, the Bligh \& Dyer method yielded a final list of 31 features in positive ion mode and 16 features in negative ion mode, while for the SPME method 39 features were identified in positive ion mode, and 24 features in negative ion mode. Relative quantification was performed by reporting fold changes between control and treated cells for each feature with significant up/down-regulation. For the given lipids, the obtained P-values were calculated using an unpaired t-test comparison of group means for each ion that yielded P-values between $8.90 \times 10^{-3}$ to $1.06 \times 10^{-25}$. Table S-2 and S-3 in Appendix summarizes the tentative identification of top lipids found in positive and negative ESI modes, using both SPME and Bligh \& Dyer methods. The similarity of the obtained list of lipid ions by Bligh \& Dyer and that of SPME indicates that these two extraction methods are comparable. Although the most dominant species were the same in both lists, clear differences were apparent in the less abundant components. Through application of the SPME method, a distinct pattern of diversity between lipid categories and sub-categories could be observed. For instance, less abundant phospholipid species such as LysoPC, LysoPE, PS, PG, DG, and CE were detected by SPME, while these features were apparently overlooked with the use of the Bligh \& Dyer method (Table S-2 and S-3 in the Appendix). On the other hand, the Bligh \& Dyer was shown to be more efficient for the detection of triglycerides and membrane phospholipids. Furthermore, according to our results, application of either method represents a sharp distinction of unsaturation and elongation in the lipids fatty acyl moieties. Based on the current results, diversity of TGs with higher degree of unsaturation was clearly detected in the treated cells using both extraction methods. This observation could also be explained by the fact that triacylglycerides provide a reservoir of fatty acids that can be mobilized for energy generation through the action of a series of lipases [51]. In this regard, it is possible that enhanced storage of triacylglycerides could be beneficial for cancer cells, as they may be used as a readily available fuel source after re-oxygenation. The PCA score plots displayed clear separation between different groups of samples (Figure S-5 in the Appendix).

\subsection{Control vs. Treated Cells: Down-regulated lipids}

By considering the lipid species, which were statistically down-regulated in the treated cells, both methods presented a list of 19 features in positive ion mode. Table S-4 in the Appendix summarizes the tentative identification of important down-regulated lipids found in positive ESI modes using both SPME and Bligh \& Dyer methods. Based on the list, it could be seen that 
down-regulated lipids are characterized by high saturation. It can be also noticed that the control cancer cells produced phospholipids and glycerolipids with shorter fatty acyl moieties. In comparison to Bligh \& Dyer, SPME provided more comprehensive simultaneous coverage of less hydrophobic lipid species with lower abundance, while Bligh \& Dyer performed well for the coverage of PC and PE as membrane phospholipids species with diversity in their aliphatic chain.

\subsection{Biological interpretation of the data}

The importance of membrane synthesis in cancer cells has been highlighted by the excess expression and activity of the enzymes that are involved in the synthesis of phosphatidylcholine (PC) and phosphatidylethanolamine (PE) [52,53]. As mammalian cells are inefficient in synthesizing polyunsaturated fatty acids because of $\Delta 3$-desaturase enzyme deficiency, a high rate of de novo lipid synthesis in tumour elevates the relative amount of saturated and monounsaturated fatty acids. In support of this hypothesis, the same group of lipids (containing short chain saturated fatty acyls) was detected more abundantly in the control cells and diminished as a result of treatment with a highly unsaturated long chain omega-3 fatty acid, leading to production of lipid species with longer aliphatic chains and multiple double bonds in their structure.

The previously published studies mainly focused on the effect of PUFAs on cell proliferation and metabolism pathways $[1,3,54]$ and not thoroughly investigated the modification of the whole lipidome profile of cancer cells when exposed to polyunsaturated fatty acids. In other work, it has been reported that cancer cells supplemented by EPA demonstrate morphological features of cell death characterized by cell shrinkage and detachment. This phenomenon could be possibly explained according to the results of the present study, in that the significantly increased number of polyunsaturated phospholipids may prevent orderly collocation and tight packing of these molecules into a neat lipid bilayer assembly, thus facilitating membrane deformation [55]. Relying solely on the obtained data, a conclusion cannot be made that increased levels of polyunsaturated lipids (including those phosphatidylcholines and phosphatidylethanolamines) in treated cells could be directly the cause of lipotoxicity and cell apoptosis. However, recently published research suggests that plasma membranes containing abundant quantities of polyunsaturated phospholipids are more prone to deformation and fission [55]. It has been previously reported that the cells supplemented with omega-3 fatty acids significantly accumulate PUFAs in triglyceride form in their cytoplasm, which can be seen as lipid droplets $[1,43]$. Enhanced lipid peroxidation, in turn, results in accumulation of toxic lipid peroxide products in cells that eventually cause cell death. Nevertheless, in spite of 
evidence revealing the tumoricidal action of various PUFAs, their exact mechanism(s) of action is still not clearly understood $[3,43,56-58]$. To this extent, our findings, taken together with other works, support the necessity of more profound investigations on the potential pathophysiological roles of this class of lipids on cancer cell metabolism. In addition, our data also supports the necessity of further chemotaxonomical investigations in this arena.

\section{Conclusion}

In the presented study solid phase microextraction was used for the first time for cellular untargeted lipidome studies using human hepatocarcinoma cells as a model. The extraction coverage, method precision and matrix effect were compared against Bligh \& Dyer protocol. Based on this preliminary study it can be seen that the proposed protocol is simple, provides good clean-up, prevents matrix effects, which is one of the main drawbacks of standard procedures and offers extraction of broad range of lipids including the less abundant species due to the equilibrium-based nature of the method. Comparison of the putatively identified compounds extracted with both methods exhibited that SPME provided more comprehensive coverage of less hydrophobic lipid species with lower abundance. Optimization of coating composition will lead to better extraction of more polar lipid components as it has been previously demonstrated for metabolomics studies [45]. On the other hand, it should be emphasized, that SPME analysis of membrane lipids and species which are not present in free form would require additional step enabling their release to the balk of the sample prior extraction, while LLE provides information on such species because they are dissolved by the solvents used in the extraction protocol. Reduced matrix effect observed for SPME approach may be of particular importance in studies, where quantitation of certain lipids will be performed i.e. in determination of lipid biomarker level. Although the studies presented here are preliminary and development of final protocol requires further investigation which would allow to understand mechanisms and influence of all variables occurring during lipid extraction with SPME, current data demonstrates a great potential of the method in monitoring of biological changes of lipidome.

\section{Notes}

The authors declare no competing financial interest.

\section{ACKNOWLEDGMENTS}


The authors thank Natural Sciences and Engineering Research Council of Canada (NSERC) for financial support. The authors also like to express their sincere gratitude to Deeptee Seebun for cell culturing and Vincent Bessonneau for his contribution in data processing.

\section{REFERENCES}

[1] K. Lim, C. Han, Y. Dai, M. Shen, T. Wu, Omega-3 polyunsaturated fatty acids inhibit hepatocellular carcinoma cell growth through blocking beta-catenin and cyclooxygenase-2, Mol. Cancer Ther. 8 (2009) 3046-3055.

[2] R. A. Siddiqui, K. Harvey, W. Stillwell, Anticancer properties of oxidation products of docosahexaenoic acid, Chem. Phys. Lipids 153 (2008) 47-56.

[3] B. Chénais, V. Blanckaert, The Janus face of lipids in human breast cancer: how polyunsaturated Fatty acids affect tumor cell hallmarks, Int. J. Breast Cancer 2012 (2012) ID 712536.

[4] R. S. Pardini, Nutritional intervention with omega-3 fatty acids enhances tumor response to antineoplastic agents, Chem. Biol. Interact. 162 (2006) 89-105.

[5] M. R. Wenk, The emerging field of lipidomics, Nat. Rev. Drug Discov. 4 (2005) 594-610.

[6] K. Sandra, P. Sandra, Lipidomics from an analytical perspective, Curr. Opin. Chem. Biol. 17 (2013) 847-853.

[7] C. C. Teo, W. P. K. Chong, E. Tan, N. B. Basri, Z. J. Low, Y. S. Ho, Advances in sample preparation and analytical techniques for lipidomics study of clinical samples, TrAC Trends Anal. Chem. 66 (2015) 1-18.

[8] A. Scalbert, L. Brennan, O. Fiehn, T. Hankemeier, B. S. Kristal, B. van Ommen, E. Pujos-Guillot, E. Verheij, D. Wishart, S. Wopereis, Mass-spectrometry-based metabolomics: limitations and recommendations for future progress with particular focus on nutrition research, Metabolomics 5 (2009) 435-458.

[9] E. G. Bligh, W. J. Dyer, A rapid method of total lipid extraction and purification, Can. J. Biochem. Physiol. 37 (1959) 911-917.

[10]J. Folch, M. Lees, G. H. Sloane Stanley, A simple method for the isolation and purification of total lipides from animal tissues, J. Biol. Chem. 226 (1957) 497-509.

[11]M. Ståhlman, C. S. Ejsing, K. Tarasov, J. Perman, J. Borén, K. Ekroos, High-throughput shotgun lipidomics by quadrupole time-of-flight mass spectrometry, J. Chromatogr. B. Analyt. Technol. Biomed. Life Sci. 877 (2009) 2664-2672.

[12]V. Matyash, G. Liebisch, T. V Kurzchalia, A. Shevchenko, D. Schwudke, Lipid extraction by methyltert-butyl ether for high-throughput lipidomics, J. Lipid Res. 49 (2008) 1137-1146.

[13]S. K. Abbott, A. M. Jenner, T. W. Mitchell, S. H. J. Brown, G. M. Halliday, B. Garner, An improved high-throughput lipid extraction method for the analysis of human brain lipids, Lipids 48 (2013) 307-318. 
[14]W. Chen, C. Zhang, L. Song, M. Sommerfeld, Q. Hu, A high throughput Nile red method for quantitative measurement of neutral lipids in microalgae, J. Microbiol. Methods 77 (2009) 4147.

[15]L. Löfgren, M. Ståhlman, G.-B. Forsberg, S. Saarinen, R. Nilsson, G. I. Hansson, The BUME method: a novel automated chloroform-free 96-well total lipid extraction method for blood plasma, J. Lipid Res. 53 (2012) 1690-1700.

[16]L. Hodson, C. M. Skeaff, B. A. Fielding, Fatty acid composition of adipose tissue and blood in humans and its use as a biomarker of dietary intake, Prog. Lipid Res. 47 (2008) 348-380.

[17]L. Y. Wang, K. Summerhill, C. Rodriguez-Canas, I. Mather, P. Patel, M. Eiden, S. Young, N. G. Forouhi, A. Koulman, Development and validation of a robust automated analysis of plasma phospholipid fatty acids for metabolic phenotyping of large epidemiological studies, Genome Med. 5 (2013) 39.

[18]L. Kortz, J. Dorow, S. Becker, J. Thiery, U. Ceglarek, Fast liquid chromatography-quadrupole linear ion trap-mass spectrometry analysis of polyunsaturated fatty acids and eicosanoids in human plasma, J. Chromatogr. B. Analyt. Technol. Biomed. Life Sci. 927 (2013) 209-213.

[19]T. Kopf, G. Schmitz, J. Chromatogr. B. Analyt. Technol. Biomed. Life Sci. Analysis of non-esterified fatty acids in human samples by solid-phase-extraction and gas chromatography/mass spectrometry, 938 (2013) 22-26.

[20]H. Y. Kim, N. Salem, Separation of lipid classes by solid phase extraction, J. Lipid Res., 31 (1990) 2285-2289.

[21]G. C. Burdge, P. Wright, A. E. Jones, S. A. Wootton, A method for separation of phosphatidylcholine, triacylglycerol, non-esterified fatty acids and cholesterol esters from plasma by solid-phase extraction, Br. J. Nutr. 84 (2000) 781-787.

[22]Separation of Lipids by Solid Phase Extraction (SPE) : Protocol Exchange http://www.nature.com/protocolexchange/protocols/2156 (accessed Aug 28, 2015).

[23]H. Kim, N. Salem, Separation of lipid classes by solid phase extractionJ. Lipid Res. 31 (1990) 2285-2289.

[24]S. Tulipani, R. Llorach, M. Urpi-Sarda, C. Andres-Lacueva, Comparative analysis of sample preparation methods to handle the complexity of the blood fluid metabolome: when less is more, Anal. Chem. 85 (2013) 341-348.

[25]C. Bylda, R. Thiele, U. Kobold, D. A. Volmer, Recent advances in sample preparation techniques to overcome difficulties encountered during quantitative analysis of small molecules from biofluids using LC-MS/MS, Analyst 139 (2014) 2265-2276.

[26]K. Nilsson, M. Andersson, O. Beck, Phospholipid removal combined with a semi-automated 96well SPE application for determination of budesonide in human plasma with LC-MS/MS, J. Chromatogr. B. Analyt. Technol. Biomed. Life Sci. 970 (2014) 31-35.

[27]Y. Yang, C. Cruickshank, M. Armstrong, S. Mahaffey, R. Reisdorph, N. Reisdorph, New sample 
preparation approach for mass spectrometry-based profiling of plasma results in improved coverage of metabolome, J. Chromatogr. A 1300 (2013) 217-226.

[28]A. P. Birjandi, F. S. Mirnaghi, B. Bojko, M. Wąsowicz, J. Pawliszyn, Application of solid phase microextraction for quantitation of polyunsaturated fatty acids in biological fluids, Anal. Chem. 86 (2014) 12022-12029.

[29]E. Cudjoe, D. Vuckovic, D. Hein, J. Pawliszyn, Investigation of the effect of the extraction phase geometry on the performance of automated solid-phase microextraction, Anal. Chem. 81 (2009) 4226-4232.

[30]E. Boyacı, K. Goryński, Á. Rodríguez-Lafuente, B. Bojko, J. Pawliszyn, Introduction of solid-phase microextraction as a high-throughput sample preparation tool in laboratory analysis of prohibited substances, Anal. Chim. Acta 809 (2014) 69-81.

[31]V. Bessonneau, B. Bojko, A. Azad, S. Keshavjee, S. Azad, J. Pawliszyn, Determination of bronchoalveolar lavage bile acids by solid phase microextraction liquid chromatography-tandem mass spectrometry in combination with metabolite profiling: comparison with enzymatic assay, J. Chromatogr. A 1367 (2014) 33-38.

[32]F. S. Mirnaghi, J. Pawliszyn, Reusable solid-phase microextraction coating for direct immersion whole-blood analysis and extracted blood spot sampling coupled with liquid chromatographytandem mass spectrometry and direct analysis in real time-tandem mass spectrometry, Anal. Chem. 84 (2012) 8301-8309.

[33]E. Boyacı, J. Pawliszyn, Micelle assisted thin-film solid phase microextraction: a new approach for determination of quaternary ammonium compounds in environmental samples, Anal Chem. 86 (2014) 8916-8921.

[34]E. Boyacı, C. Sparham, J. Pawliszyn, Thin-film microextraction coupled to LC-ESI-MS/MS for determination of quaternary ammonium compounds in water samples, Anal. Bioanal. Chem. 406 (2014) 409-420.

[35]O. P. Togunde, E. Cudjoe, K. D. Oakes, F. S. Mirnaghi, M. R. Servos, J. Pawliszyn, Determination of selected pharmaceutical residues in wastewater using an automated open bed solid phase microextraction system, J. Chromatogr. A 1262 (2012) 34-42.

[36]F. S. Mirnaghi, F. Mousavi, S. M. Rocha, J. Pawliszyn, Automated determination of phenolic compounds in wine, berry, and grape samples using 96-blade solid phase microextraction system coupled with liquid chromatography-tandem mass spectrometry, J. Chromatogr. A 1276 (2013) 12-19.

[37]F. S. Mirnaghi, D. Hein, J. Pawliszyn, Thin-Film Microextraction Coupled with Mass Spectrometry and Liquid Chromatography-Mass Spectrometry, Chromatographia 76 (2013) 1215-1223.

[38]D. Vuckovic, E. Cudjoe, F. M. Musteata, J. Pawliszyn, Automated solid-phase microextraction and thin-film microextraction for high-throughput analysis of biological fluids and ligand-receptor binding studies, Nat. Protoc. 5 (2010) 140-161.

[39]N. Reyes-Garcés, B. Bojko, J. Pawliszyn, High throughput quantification of prohibited substances 
in plasma using thin film solid phase microextraction, J. Chromatogr. A 1374 (2014) 40-49.

[40]M. Wąsowicz, A. Jerath, B. Bojko, V. Sharma, J. Pawliszyn, S. McCluskey, Use of a novel technique, solid phase microextraction, to measure tranexamic acid in patients undergoing cardiac surgery, Can. J. Anaesth. 59 (2012) 14-20.

[41]J. Dai, J. Shen, W. Pan, S. Shen, U. N. Das, Effects of polyunsaturated fatty acids on the growth of gastric cancer cells in vitro, Lipids Health Dis. 12 (2013) 71.

[42]S. J. Iverson, S. L. Lang, M. H. Cooper, Comparison of the Bligh and Dyer and Folch methods for total lipid determination in a broad range of marine tissue, Lipids 36 (2001) 1283-1287.

[43]F. S. Mirnaghi, Y. Chen, L. M. Sidisky, J. Pawliszyn, Optimization of the coating procedure for a high-throughput 96-blade solid phase microextraction system coupled with LC-MS/MS for analysis of complex samples, Anal. Chem. 83 (2011) 6018-6025.

[44]G. J. Patti, R. Tautenhahn, G. Siuzdak, Meta-analysis of untargeted metabolomic data from multiple profiling experiments, Nat. Protoc. 7 (2012) 508-516.

[45]F. Mousavi, B. Bojko, J. Pawliszyn, Development of high throughput 96-blade solid phase microextraction-liquid chromatrography-mass spectrometry protocol for metabolomics, Anal. Chim. Acta 892 (2015) 95-104.

[46]E. Boyacı, Á. Rodríguez-Lafuente, K. Goryński, F. Mirnaghi, É. A. Souza-Silva, D. Hein, J. Pawliszyn, Sample preparation with solid phase microextraction and exhaustive extraction approaches: Comparison for challenging cases, Anal. Chim. Acta 873 (2014) 14-30.

[47]Food and Drug Administration, Department of Health and Human Services. Fed. Regist 2001, 66, 28526-28527.

[48]T. Houjou, K. Yamatani, M. Imagawa, T. Shimizu, R. Taguchi, A shotgun tandem mass spectrometric analysis of phospholipids with normal-phase and/or reverse-phase liquid chromatography/electrospray ionization mass spectrometry, Rapid Commun. Mass Spectrom. 19 (2005) 654-666.

[49]G. Liebisch, B. Lieser, J. Rathenberg, W. Drobnik, G. Schmitz, High-throughput quantification of phosphatidylcholine and sphingomyelin by electrospray ionization tandem mass spectrometry coupled with isotope correction algorithm, Biochim. Biophys. Acta 1686 (2004) 108-117.

[50]B. K. Matuszewski, M. L. Constanzer, C. M. Chavez-Eng, Strategies for the assessment of matrix effect in quantitative bioanalytical methods based on HPLC-MS/MS, Anal. Chem. 75 (2003) 3019-3030.

[51]Y. Li, F. Ghasemi Naghdi, S. Garg, T. C. Adarme-Vega, K. J. Thurecht, W. A. Ghafor, S. Tannock, P. M. Schenk, A comparative study: the impact of different lipid extraction methods on current microalgal lipid research, Microb. Cell Fact. 13 (2014) 14.

[52]C. R. Santos, A. Schulze, Lipid metabolism in cancer, FEBS J. 279 (2012) 2610-2623.

[53]F. Gibellini, T. K. Smith, The Kennedy pathway-de novo synthesis of phosphatidylethanolamine and phosphatidylcholine, IUBMB Life, 62 (2010) 414-428. 
[54]M. Vinciguerra, A. Sgroi, C. Veyrat-Durebex, L. Rubbia-Brandt, L. H. Buhler, M. Foti, Unsaturated fatty acids inhibit the expression of tumor suppressor phosphatase and tensin homolog (PTEN) via microRNA-21 up-regulation in hepatocytes, Hepatology 49 (2009) 1176-1184.

[55]M. Pinot, S. Vanni, S. Pagnotta, S. Lacas-Gervais, L. -A. Payet, T. Ferreira, R. Gautier, B. Goud, B. Antonny, H. Barelli, Lipid cell biology. Polyunsaturated phospholipids facilitate membrane deformation and fission by endocytic proteins, Science 345 (2014) 693-697.

[56]M. Fukasawa, Y. Tanaka, S. Sato, Y. Ono, Y. Nitahara-Kasahara, T. Suzuki, T. Miyamura, K. Hanada, M. Nishijima, Enhancement of de novo fatty acid biosynthesis in hepatic cell line Huh7 expressing hepatitis C virus core protein, Biol. Pharm. Bull. 29 (2006) 1958-1961.

[57]T.-Y. Chi, G. G. Chen, P. B. S. Lai, Eicosapentaenoic acid induces Fas-mediated apoptosis through a p53-dependent pathway in hepatoma cells, Cancer J. 10 (2004) 190-200.

[58]S. Itoh, A. Taketomi, N. Harimoto, E. Tsujita, T. Rikimaru, K. Shirabe, M. Shimada, Y. Maehara, Antineoplastic effects of gamma linolenic Acid on hepatocellular carcinoma cell lines, J. Clin. Biochem. Nutr. 47 (2010) 81-90. 
Captions to the Figures

Figure 1. Comparison of method precision (expressed as $\%$ RSD for $n=5$ replicates) obtained for Bligh \& Dyer with methanol/chloroform and the validated SPME technique using C18 coatings

Figure 2. (a \& c) lon map ( $\mathrm{m} / \mathrm{z}$ versus retention time) for HUH7 cell samples extracted by Bligh \& Dyer and SPME. ( $b$ \& d) Number of peaks with given signal variation (\% RSD) obtained for the two independent data sets

Figure 3. PCA plot presenting clusters of data obtained for treated and control cells afterextraction with SPME and Bligh\&Dyer methods, for QC prepared from pooled aliquots of analyzed samples and for mixture of selected standards used for additional monitoring of instrument performance.

Figure 4. Example OPLS-Da models (right) SPME and (left) B\&D extraction methods comparing control and treated cell samples. Circle and triangle markers in both panels show control and treated group of cells, respectively

Figure 5. Comparison of extraction efficiency for identified Lipid Groups by (left) SPME and (right) Bligh\&Dyer in covering a broad range of class-specific lipids

Figure 6. Evaluation of matrix effect of the extraction methods; the highlighted blue area illustrates the analytical acceptable range for matrix effect 